\title{
Synthesis of giant globular multivalent glycofullerenes as potent inhibitors in a model of Ebola virus infection
}

\author{
Antonio Muñoz ${ }^{1}$, David Sigwalt', ${ }^{2,3}$, Beatriz M. Illescas ${ }^{1}$, Joanna Luczkowiak4, Laura Rodríguez-Pérez', \\ Iwona Nierengarten ${ }^{3}$, Michel Holler ${ }^{3}$, Jean-Serge Remy², Kevin Buffet ${ }^{5}$, Stéphane P. Vincent ${ }^{5}$,

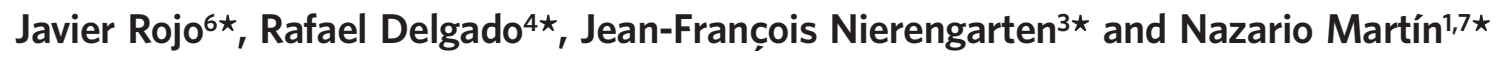

\begin{abstract}
The use of multivalent carbohydrate compounds to block cell-surface lectin receptors is a promising strategy to inhibit the entry of pathogens into cells and could lead to the discovery of novel antiviral agents. One of the main problems with this approach, however, is that it is difficult to make compounds of an adequate size and multivalency to mimic natural systems such as viruses. Hexakis adducts of [60]fullerene are useful building blocks in this regard because they maintain a globular shape at the same time as allowing control over the size and multivalency. Here we report water-soluble tridecafullerenes decorated with 120 peripheral carbohydrate subunits, so-called 'superballs', that can be synthesized efficiently from hexakis adducts of [60]fullerene in one step by using copper-catalysed azide-alkyne cycloaddition click chemistry. Infection assays show that these superballs are potent inhibitors of cell infection by an artificial Ebola virus with half-maximum inhibitory concentrations in the subnanomolar range.
\end{abstract}

M ultivalency is a general and efficient tool used by nature to achieve strong interactions in a reversible manner. At the molecular level, multivalent interactions have the advantage that they enhance drastically the binding between molecules when compared with monovalent binding ${ }^{1}$.

A remarkable example from nature in which multivalency plays a significant role is the interaction between viruses and bacteria with their respective host cells. In particular, the DC-SIGN (dendritic cell-specific intercellular adhesion molecule-3-grabbing non-integrin) receptor is one of the most important pathogen-recognition receptors. This lectin efficiently recognizes, in a multivalent manner, saccharides that contain mannoses and fucoses from glycoproteins (GPs) ${ }^{2}$. In this regard, it is well established that protein-carbohydrate interactions are a key issue in a variety of biological processes, but because the affinity of simple glycans for their respective receptors is often weak, multivalent interactions typically occur. An important challenge for research nowadays is to better understand multivalency and its practical use. In a broader sense, glycobiology is currently a field of research in which chemically inspired approaches and strategies are producing significant advances ${ }^{3}$.

It is well known, however, that some viruses are able to escape the immune defence by using DC-SIGN as an entry point to infect the cell. Therefore, to inhibit the entry of pathogens by blocking this receptor at the early stages of infection represents a valuable strategy for the design of new antiviral agents. To address this challenge, a variety of different multivalent scaffold architectures have been synthesized, all of them endowed with multiple carbohydrates ${ }^{4}$.
The design of these glycoconjugates typically requires a multivalent central scaffold or core covalently connected to the carbohydrate epitopes that decorate the periphery. Thus, glycoclusters in which the carbohydrate units are connected directly to the core, glycodendrimers connected through a dendritic structure and glycopolymers that involve a polymeric backbone have been investigated intensively in recent years ${ }^{5-9}$.

Fullerenes have also been employed as a biocompatible scaffold for the multivalent presentation of ligands, given the possibility of multiple functionalization on their convex surface. In particular, hexakis adducts of [60]fullerene with a $\mathrm{T}_{\mathrm{h}}$-symmetrical octahedral addition pattern have a unique three-dimensional structure that allows the introduction of six one-type or mixed-type addends ${ }^{10,11}$. Such derivatives can be obtained in one synthetic step by the addition of malonates to $\mathrm{C}_{60}$, but because the reaction is very sensitive to steric effects this approach has been limited by the low yields that result when larger malonates are used ${ }^{12}$. Recently, we developed a procedure based on a click-chemistry approach that provides hexakis adducts with 12 alkyne or azide terminal groups in high yields from simple malonates ${ }^{13,14}$. These hexakis adducts can be functionalized easily and efficiently by using the copper-catalysed azide-alkyne cycloaddition (CuAAC) reaction, which allows the introduction of 12 functional groups simultaneously in a regioselective and efficient way. As these hexakis adducts show an octahedral arrangement of equally separated addends located on the [60]fullerene periphery in a globular topology, they constitute a very attractive platform for the study of multivalent interactions with lectins with important advantages, such as a better biocompatibility of the carbon central core, the globular

'Departamento de Química Orgánica, Facultad de Química, Universidad Complutense, Madrid 28040, Spain. ${ }^{2}$ Laboratory V-SAT (CAMB UMR 7199, CNRS), Labex Medalis, Université de Strasbourg, 74 Route du Rhin, Illkirch-Graffenstaden 67401, France. ${ }^{3}$ Laboratoire de Chimie des Matériaux Moléculaires, Université de Strasbourg et CNRS (UMR 7509), Ecole Européenne de Chimie, Polymères et Matériaux, 25 rue Becquerel, Strasbourg 67087, France. ${ }^{4}$ Laboratorio de Microbiología Molecular, Instituto de Investigación Hospital 12 de Octubre (imas12), Madrid 28041, Spain. ${ }^{5}$ Département de Chimie, Laboratoire de Chimie Bio-Organique, University of Namur (FUNDP), rue de Bruxelles 61, Namur B-5000, Belgium. ${ }^{6}$ Glycosystems Laboratory, Instituto de Investigaciones Químicas (IIQ), CSIC - Universidad de Sevilla, Av. Américo Vespucio 49, Seville 41092, Spain. ${ }^{7}$ IMDEA-Nanoscience, Campus Cantoblanco, Madrid 28049, Spain. ^e-mail: nazmar@quim.ucm.es; javier.rojo@iiq.csic.es; rafael.delgado@salud.madrid.org; nierengarten@unistra.fr 
<smiles>CC#CCCCO</smiles><smiles>CCOC(=O)CC(=O)OCCCC#C[SiH3]</smiles>

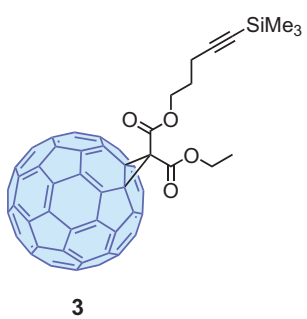

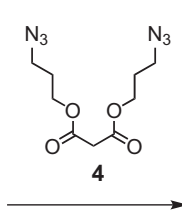

(iii)

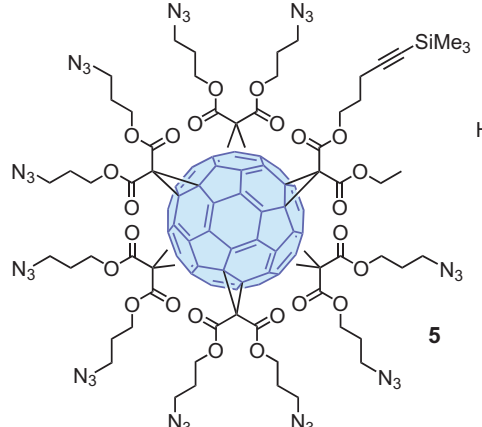

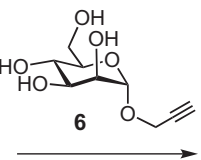

(iv)
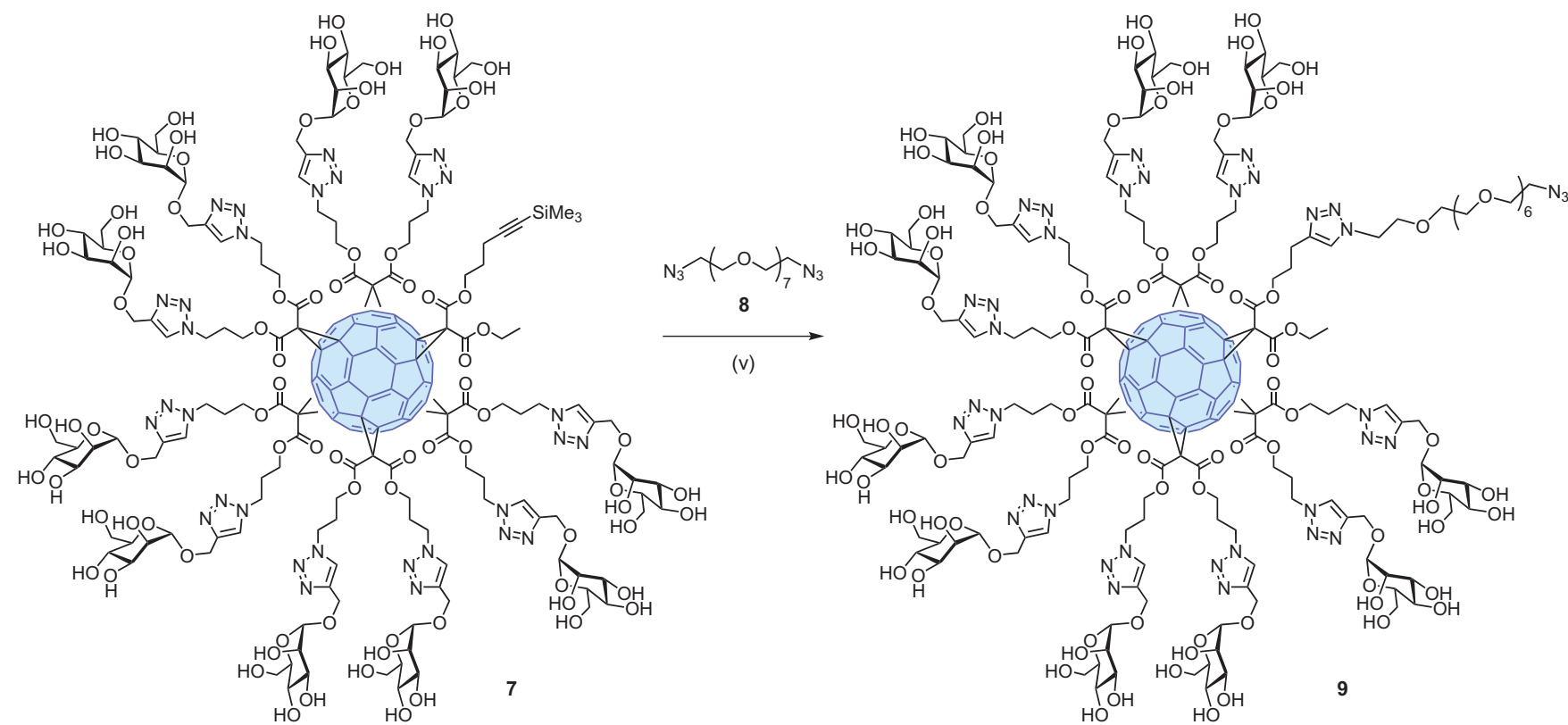

Figure 1 | Synthesis of azide-substituted glycofullerene 9 appended with a long linker and mannose as the carbohydrate unit. Five linear steps are required to prepare compound 9 in a $26 \%$ overall yield. Reagents and conditions: (i) ethylmalonyl chloride, pyridine, $\mathrm{CH}_{2} \mathrm{Cl}_{2}, 0$ to $25{ }^{\circ} \mathrm{C}$, one hour $\left(99 \%\right.$ ); (ii) $\mathrm{C}_{60}, \mathrm{I}_{2}$, $\mathrm{DBU}, 25^{\circ} \mathrm{C}$, PhMe, 16 hours (49\%); (iii) $\mathrm{CBr}_{4}, \mathrm{DBU}, 25^{\circ} \mathrm{C}, \mathrm{ODCB}, 72$ hours (67\%); (iv) $\mathrm{CuSO}_{4} \cdot 5 \mathrm{H}_{2} \mathrm{O}$, sodium ascorbate, $\mathrm{THF} / \mathrm{H}_{2} \mathrm{O}, 100{ }^{\circ} \mathrm{C}(\mathrm{MW}$ ), two hours (91\%); (v) 8 (19 equiv.), $\mathrm{CuSO}_{4} \cdot 5 \mathrm{H}_{2} \mathrm{O}$, sodium ascorbate, TBAF, THF $/ \mathrm{H}_{2} \mathrm{O}, 80{ }^{\circ} \mathrm{C}(\mathrm{MW}), 1.5 \mathrm{hours}(87 \%)$.

geometry of the functional groups and an easy and versatile chemical functionalization.

Recently, we reported the syntheses of [60]fullerene hexakis adducts endowed with different carbohydrate units in the periphery. Depending on the starting sugar derivatives employed (monomer, dimer or trimer) 12, 24 and up to 36 monosaccharides were introduced on the periphery of the fullerene central core in a straightforward manner ${ }^{14-17}$.

Some of the aforementioned glycofullerenes showed interesting biomedical applications ${ }^{18}$. A significant multivalent effect towards various glycosidases was observed in the inhibition profile of a [60]fullerene hexakis adduct endowed with 12 iminosugar units ${ }^{16}$. However, fullerene hexakis adducts that bear 12 mannoses on the periphery behave as inhibitors of FimH, a bacterial adhesin ${ }^{19}$. Furthermore, studies carried out on hexakis adducts of [60]fullerene endowed with 12, 24 and 36 mannoses have shown a multivalent effect in the interaction with concanavalin $\mathrm{A}^{15}$, and act as efficient inhibitors of cell infection by Ebola-pseudotyped viral particles ${ }^{20}$. These preliminary studies reveal that fullerenes are adequate platforms for the multivalent presentation of carbohydrates, and thus pave the way to the covalent linkage of a wide variety of different bioactive molecules in a multivalent manner and to a singular and less-explored globular topology.

In this work, we synthesized molecules with a globular topology formed by making hexakis adducts of [60]fullerene (with the fastest dendrimeric growth reported up to now) through the introduction of 120 sugar units in one synthetic step by using the highly efficient CuAAC click-chemistry methodology. The synthesis of hexakis adducts of [60]fullerene with an octahedral addition pattern has been reported previously. This previous example used macrocyclic bismalonates and enabled the synthesis of heptafullerenes with tunable properties ${ }^{21-23}$. In contrast, our click-chemistry approach yields tridecafullerenes in which the central [60]fullerene is covalently connected to 12 [60]fullerenes, each endowed, in turn, with ten monosaccharides. To confirm the proposed structures, we carried out a thorough structural study that involved a variety of techniques (infrared, ${ }^{1} \mathrm{H}$ NMR and ${ }^{13} \mathrm{C}$ NMR spectroscopy, dynamic light scattering (DLS), transmission electron microscopy (TEM) and X-ray photoelectron spectroscopy (XPS)). Biological studies revealed that the new giant glycofullerenes exhibit a very strong inhibition of cell infection by Ebola-pseudotyped viral particles with half-maximum inhibitory concentration $\left(\mathrm{IC}_{50}\right)$ values in the subnanomolar range.

\section{Results and discussion}

Synthesis. The synthesis of the glycofullerene superballs was carried out as depicted in Figs 1-3. Although, at first glance, the molecules $17 \mathrm{a}-17 \mathrm{c}$ may appear difficult to obtain, mainly because of their size and molecular complexity, their synthesis is straightforward and, most importantly, easily reproducible, and it affords the final 


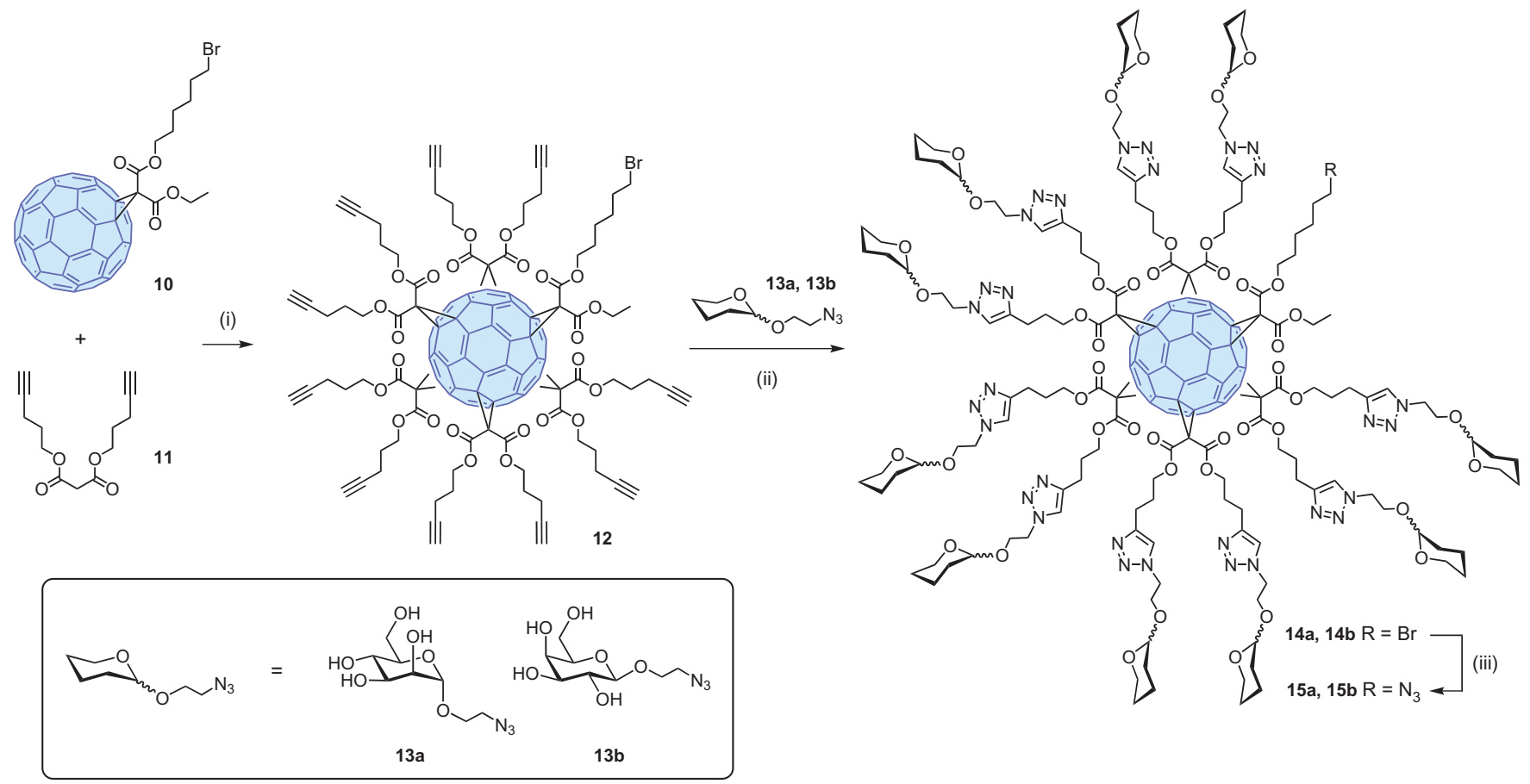

Figure 2 | Synthetic pathway to azide-substituted glycofullerenes 15a and 15b with a short spacer. The peripheral glycofullerene units $15 \mathrm{a}$ and $15 \mathrm{~b}$ were prepared using Bingel conditions and CuAAC click chemistry to conjugate the carbohydrate moieties. Reagents and conditions: (i) $\mathrm{CBr}{ }_{4}, \mathrm{DBU}, 2 \mathrm{o}^{\circ} \mathrm{C}, \mathrm{PhMe}$, 72 hours (49\%); (ii) 2-azidoethyl $\alpha$-D-mannopyranoside for 14 a (or 2-azidoethyl $\alpha$-D-galactopyranoside for $14 b$ ), $\mathrm{CuBr}^{\mathrm{S}} \mathrm{S}\left(\mathrm{CH}_{3}\right)_{2}$, sodium ascorbate, $\mathrm{Cu}^{0}$, DMSO, 72 hours (14a, 86\%; 14b, 86\%); (iii) $\mathrm{NaN}_{3}, 70^{\circ} \mathrm{C}(\mathrm{MW})$, DMSO, three hours (15a, 84\%; 15b, 81\%).

compounds in good overall yields. Furthermore, as discussed below, purification of the samples-which includes the required removal of copper for further biological studies-is carried out easily because, after column filtration, compounds $17 \mathrm{a}$ and $17 \mathrm{~b}$ are precipitated in the reaction medium and pure samples that show highly reproducible biological assays are obtained.

To study the effect of the size and/or steric congestion of these superballs on biological properties, two different and complementary synthetic strategies were developed simultaneously to obtain final products with different spacers between the central fullerene core and the peripheral carbohydrate-substituted fullerene appendages. In both cases, the synthesis relies on the grafting of clickable $\mathrm{A}_{10} \mathrm{~B}$ macromonomers onto a compact $\mathrm{C}_{60}$ hexa-adduct scaffold that bears 12 terminal alkyne units. The preparation of macromonomer 9 is depicted in Fig. 1. Esterification of alcohol 1 with ethylmalonyl chloride followed by reaction of the resulting malonate (2) with $\mathrm{C}_{60}$ under Bingel conditions gave methanofullerene 3 . Subsequent treatment of 3 with an excess of malonate $4, \mathrm{CBr}_{4}$ and 1,8-diazabicyclo[5.4.0] undec-7-ene (DBU) in $o$-dichorobenzene (ODCB) gave building block 5 to which the mannose derivative 6 was clicked. Importantly, the trimethylsilyl-protected alkyne unit is not reactive under these conditions and intermediate 7 was thus obtained in a good yield. Finally, compound 7 was desilylated in situ with tetrabutylammonium fluoride (TBAF) to generate the corresponding terminal alkyne, and reaction with a large excess of diazide 8 provided the desired macromonomer 9 in $87 \%$ yield.

The synthesis of macromonomers $15 a$ and $15 b$ starts from monoadduct 10, which results from the Bingel nucleophilic cyclopropanation of $\mathrm{C}_{60}$ with 6-bromohexyl ethyl malonate (Fig. 2) ${ }^{24}$. To obtain the [5:1]-hexa-adduct 12, a tenfold excess of di(pent-4-yn-1-yl) malonate and a $\sim 50$-fold excess of carbon tetrabromide were added in the presence of DBU as the base. Flashchromatography purification provided hexa-adduct 12 as a red solid. Different carbohydrate azides (mannose, galactose) were then linked to $\mathrm{C}_{60}$ by the $\mathrm{CuAAC}$ reaction, with $\mathrm{CuBr} \cdot \mathrm{S}\left(\mathrm{CH}_{3}\right)_{2}$ as the catalyst and sodium ascorbate as the reducing agent in the presence of a piece of metallic $\mathrm{Cu}$, to yield derivatives $14 \mathrm{a}$ and $14 \mathrm{~b}$. The nucleophilic substitution of bromine by the azide was carried out with an excess of sodium azide under microwave (MW) heating, and thus gave the versatile building blocks 15a and 15b.

The azide-containing macromonomers $9,14 \mathrm{a}$ and $14 \mathrm{~b}$ were then clicked to the symmetric alkyne derivative $16^{14}$ under CuAAC conditions. Superballs $17 \mathrm{a}-17 \mathrm{c}$ substituted with up to 120 monosaccharide units were thus obtained with yields over 70\% (Fig. 3). Derivatives $17 \mathrm{a}$ and $\mathbf{1 7 b}$ were obtained in only three synthetic steps from easily accessible starting materials and the use of protecting groups was avoided.

Although the three tridecafullerenes $17 a-17 c$ contain the same number of monosaccharides, compound $17 \mathrm{c}$ has a larger spacer between the central fullerene moiety and the peripheral carbohydrate-functionalized fullerenes. This longer spacer affects the flexibility and the size of the compound and can favour the accessibility and availability of the carbohydrate ligands to interact with the receptor, which could have an important influence on the biological activity ${ }^{20}$.

However, the aforementioned synthetic approaches enable fullerene derivatives to be synthesized from malonates endowed with alkyl chains of variable length through a Bingel cyclopropanation followed by a subsequent CuAAC reaction. This synthetic strategy affords hexakis adducts with long chains that connect the fullerenes and carbohydrates whereas their direct preparation from suitably functionalized malonates that bear long chains typically occurs with low yields ${ }^{10}$.

Characterization of these superballs was carried out by standard spectroscopic techniques. Thus, Fourier transform infrared (FTIR) spectra do not show the presence of either of the azide groups (the typical signal is observed at $\sim 2,097 \mathrm{~cm}^{-1}$ ) or the alkynes $\left(\sim 2,117 \mathrm{~cm}^{-1}\right)$ (see the Supplementary Information). The molecular ion peak of these compounds could not be detected, but the transfer of glycoclusters of such high molecular weights into the gas 


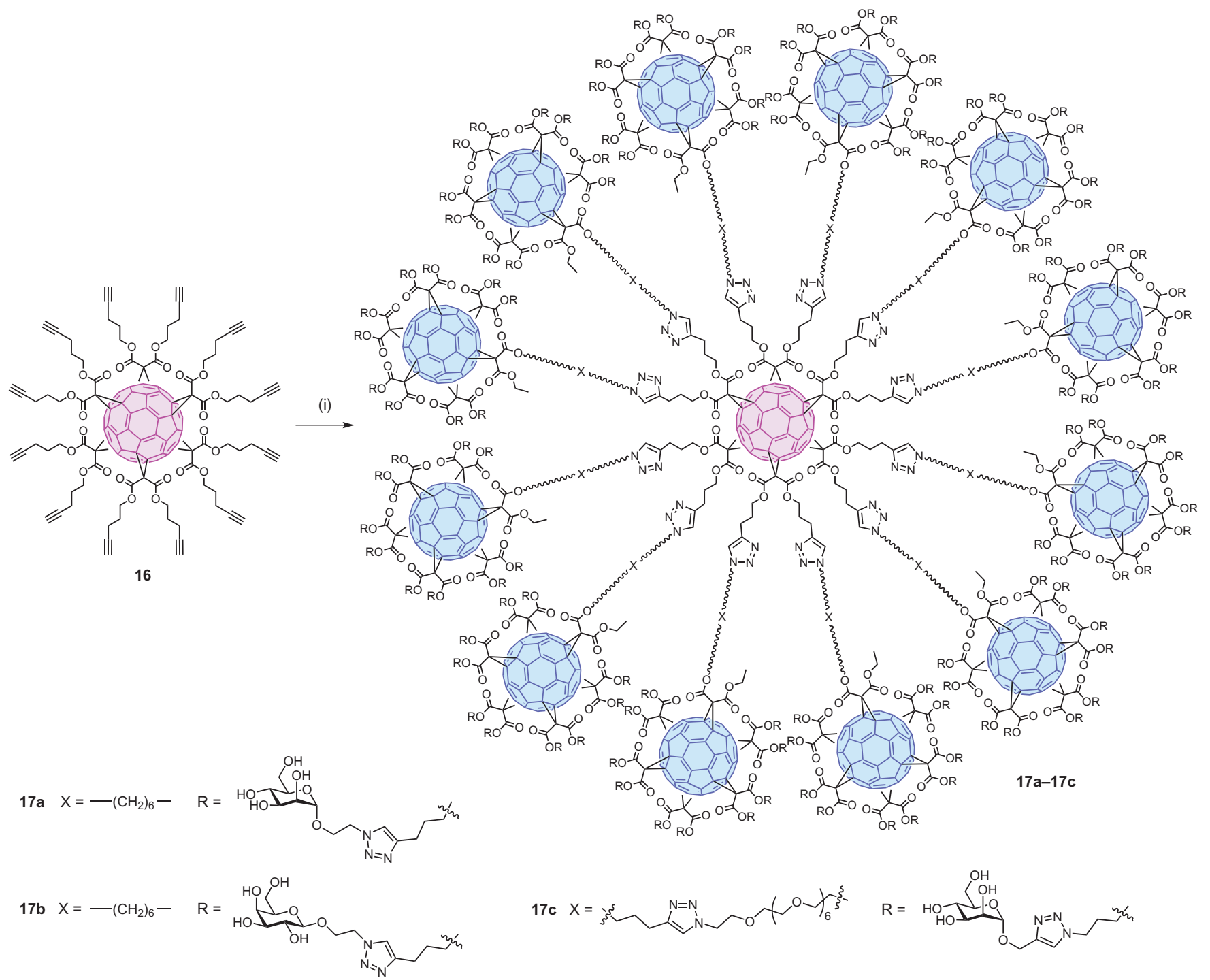

Figure 3 | Syntheses of the tridecafullerenes 17a-17c using CuAAC click chemistry. The core fullerene 16 (endowed with 12 alkyne groups) is joined to the peripheral fullerene-based compounds $9,15 \mathrm{a}$ and $15 \mathrm{~b}$ by click chemistry. Reagents and conditions: for compounds $17 \mathrm{a}$ or $17 \mathrm{~b}$ (i) $15 \mathrm{a}$ or $15 \mathrm{~b}, \mathrm{CuBr}^{\mathrm{S}} \mathrm{S}\left(\mathrm{CH}_{3}\right)_{2}$, sodium ascorbate, $\mathrm{Cu}(0), \mathrm{DMSO}, 25^{\circ} \mathrm{C}, 48$ hours (17a (from 15a), 73\%; 17b (from 15b), 79\%); for compound 17c (i) $9, \mathrm{CuSO}_{4} \cdot 5 \mathrm{H}_{2} \mathrm{O}, \mathrm{sodium}$ ascorbate, $\mathrm{THF} / \mathrm{H}_{2} \mathrm{O}, 80^{\circ} \mathrm{C}(\mathrm{MW})$, two hours (76\%).

phase during matrix-assisted laser desorption/ionization-time-offlight mass spectroscopy analysis is very difficult. Moreover, both the sugars and the fullerene hexa-adduct moieties give rise to a high level of fragmentation ${ }^{25}$. The unambiguous structural characterization of $17 \mathrm{a}-17 \mathrm{c}$ was, however, greatly facilitated by their high symmetry. Indeed, ${ }^{13} \mathrm{C}$ NMR spectroscopy was particularly helpful for the characterization of hexakis adducts of [60]fullerene, as only two signals are usually observed for the $s p^{2}$ carbons of $\mathrm{C}_{60}$, which provides evidence for the octahedral symmetry of the fullerene core. As a typical example, the ${ }^{13} \mathrm{C}$ NMR spectrum of compound $17 \mathrm{a}$ is depicted in Fig. 4. In this case, only two $s p^{2}$ carbons are observed in the spectra $(\delta \approx 145.4$ and $141.6 \mathrm{ppm})$, in which we can also distinguish the two different kinds of triazole rings present in the compound (at $\delta \approx 146.9$ and $123.3 \mathrm{ppm}$ for the carbons of the outer triazole rings and at $\delta \approx 146.4$ and $122.9 \mathrm{ppm}$ for the carbons of the inner triazole rings). In addition, only one signal is detected for all the carbonyl groups $(\delta \approx 163.8 \mathrm{ppm})$ and the $s p^{3}$ carbons of the $\mathrm{C}_{60}(\delta \approx 69.3 \mathrm{ppm})$, whereas the malonate bridgehead carbons present in the structure are observed at $\delta \approx 45.2 \mathrm{ppm}$.

Additional characterization was achieved by DLS analysis $\left(\mathrm{H}_{2} \mathrm{O}\right.$, $0.01 \mathrm{mg} \mathrm{ml}^{-1}$ and $0.1 \mathrm{mg} \mathrm{ml}^{-1}$ ), in which, regardless of the concentration used, we found two or three main size distributions for $17 \mathbf{a}-17 \mathrm{c}$ (Supplementary Fig. 1). This is compatible with a weak aggregation of $17 \mathrm{a}-17 \mathrm{c}$ in water. The first, at around 5-6 nm, must correspond to only one molecule, whereas the second, at $\sim 120-150 \mathrm{~nm}$, and the third, at $\geq 200 \mathrm{~nm}$, show the aggregation of several molecules. In dimethylsulfoxide (DMSO) $\left(0.1 \mathrm{mg} \mathrm{ml^{-1 } )}\right.$ (Supplementary Fig. 2), although most of the molecules show no aggregation, the presence of aggregates of different sizes and, especially, of very large aggregates is also detected. The tendency to form aggregates was also confirmed by the broadening of the ${ }^{13} \mathrm{C}$ NMR spectrum recorded in $\mathrm{D}_{2} \mathrm{O}$ when compared with that recorded in DMSO- $d_{6}$ (see the Supplementary Information).

The TEM images of freshly prepared samples reveal the presence of small spherical particles, which correspond to a few or even just one molecule $(\sim 4 \mathrm{~nm})$, independently of whether the concentration is $0.01 \mathrm{mg} \mathrm{ml}^{-1}$ or $0.1 \mathrm{mg} \mathrm{ml}^{-1}$, in good agreement with the experimental findings in DLS analyses (Fig. 5 and Supplementary Fig. 3).

XPS was additionally used to confirm the composition of the sugar balls. This surface technique enables the identification of the atoms present on the molecule together with their chemical state and their relative abundance. The survey spectra of superball 


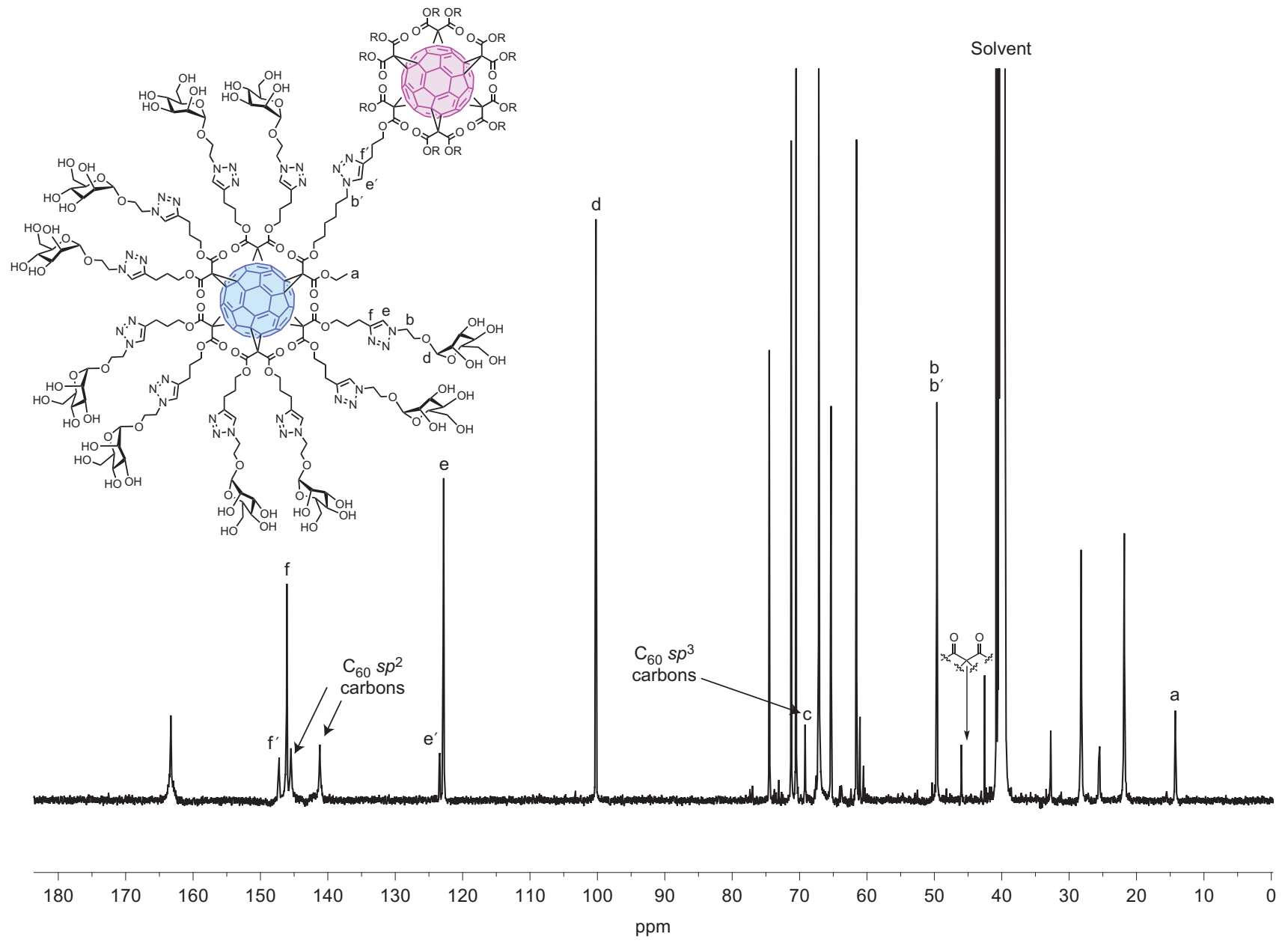

Figure 4 | ${ }^{13} \mathrm{C}$ NMR spectrum of tridecafullerene 17 a in DMSO- $d_{6}$. The assignments depict the most representative signals. This spectrum is in full agreement with the T-symmetrical structure of the compound and enables confirmation of the complete functionalization of the alkyne moieties in precursor 16 because of the absence of signals that correspond to the sp-hybridized carbons of the alkyne groups.

17a (Supplementary Fig. 4) displays the $\mathrm{C} 1 s, \mathrm{O} 1 s$ and $\mathrm{N} 1 s$ features as expected, with no additional spectroscopy signatures of possible impurities. Moreover, the high-resolution N $1 s$ core-level spectrum (Supplementary Fig. 4, inset right) was composed of two different components with a 1:2 ratio of the integrated areas; that located at $400.3 \mathrm{eV}$ is related to one nitrogen atom of the triazole ring $(\mathrm{N}-\mathrm{N}-\mathrm{N})$ and the other, centred at $398.8 \mathrm{eV}$, is attributed to the other two nitrogen atoms attached to carbon atoms $(\mathrm{C}-\mathrm{N})^{26}$. The absence of a well-resolved peak around $405.0 \mathrm{eV}$ demonstrates the lack of the electron-deficient nitrogen of the azide group in the final compound ${ }^{27,28}$. The composition of compounds $17 \mathrm{~b}$ and $17 \mathrm{c}$ was also ascertained by their respective XPS analyses, which showed the presence of the expected elements according to their relative abundance (Supplementary Fig. 4 and Supplementary Table 1).

Biological studies. A number of molecules, including DC-SIGN (dendritic cell-specific intercellular adhesion molecule-3-grabbing non-integrin), have been proposed as receptors for the Ebola virus $(\mathrm{EBOV})^{29-31}$. Although DC-SIGN is not the main receptor in the case of the EBOV, it is thought to play a significant role in the cell entrance of this infectious agent in significant cell populations, such as dendritic cells ${ }^{29,32}$, and thus facilitate early viral dissemination. Therefore, DC-SIGN can function as a good model for (1) studying the first steps of pathogenesis of EBOV and (2) screening the antiviral strategies based on DC-SIGN-targeting compounds for prevention and treatment purposes. DC-SIGN recognizes mannosylated and fucosylated oligosaccharides presented in a multivalent manner on the surface of several pathogen envelope GPs. Thus, the preparation of multivalent carbohydrate systems is necessary for an efficient interaction with this receptor as well as for effective competition with the natural ligands. In this study we evaluated the inhibitory effect of giant globular multivalent glycofullerenes in an experiment with the direct infection of Jurkat cells that express the surface receptor DC-SIGN (Jurkat-DC-SIGN) with pseudotyped viral particles that present EBOV-GP. These globular multivalent systems are water soluble and show no cytotoxicity in cell lines, which allows the study of their potential biological function in preventing viral infection. All the multivalent compounds were checked for the possibility of blocking the DCSIGN receptor in six independent experiments. The results of blocking the DC-SIGN receptor by different compounds are reported as a function of concentration. A 50\% inhibition of the infection was calculated with a $95 \%$ confidence interval (CI). As a control, infection with DC-SIGN-independent vesicular stomatitis virus envelope GP (VSV-GP)-pseudotyped lentiviral particles ${ }^{29}$ was performed under the same conditions.

The results obtained in the infection experiment revealed the dependence of the inhibition effect on mannoses. Compound $\mathbf{1 7 b}$, which displayed 120 galactoses, as expected, was not able to inhibit the infection process mediated by DC-SIGN. Compounds with 120 mannose-based residues (17a and $17 \mathrm{c}$ ) showed very strong antiviral activity at picomolar to nanomolar concentrations. Compound 17 a could effectively block EBOV infection at 

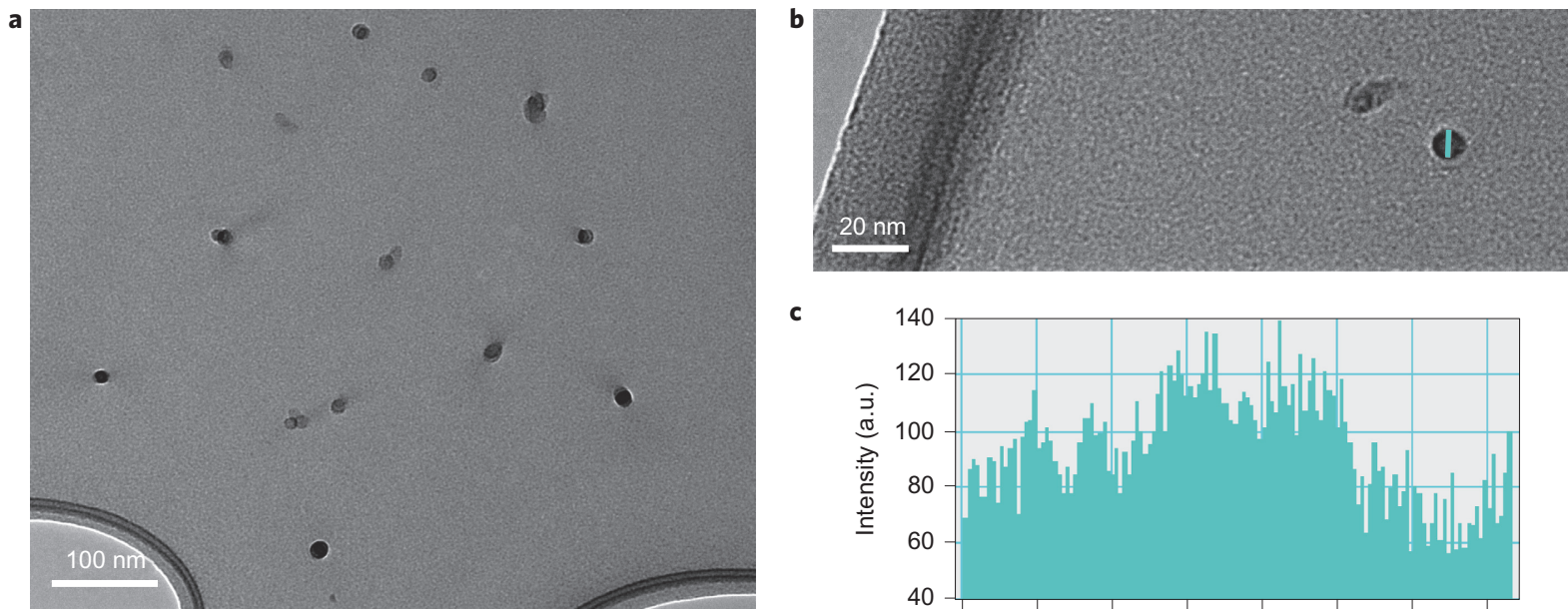

C

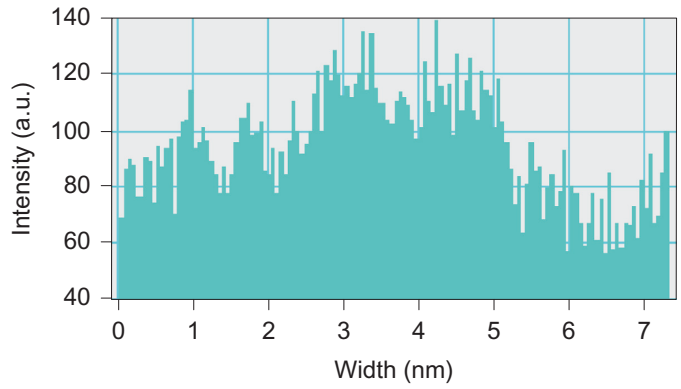

Figure 5 | TEM images and DLS analysis of tridecafullerene 17a. These images show small spherical particles with a diameter of around $4 \mathrm{~nm}$, which corresponds to a single molecule. $\mathbf{a}$, TEM images of compound 17a on deposition of a $0.01 \mathrm{mg} \mathrm{ml}^{-1}$ solution in $\mathrm{H}_{2} \mathrm{O}$. b. Detail of a particle that apparently corresponds to one molecule. $\mathbf{c}$. Width profile of the particle shown in $\mathbf{b}$, which has a diameter of $\sim 4 \mathrm{~nm}$ in accordance with the DLS data. a.u., arbitrary units

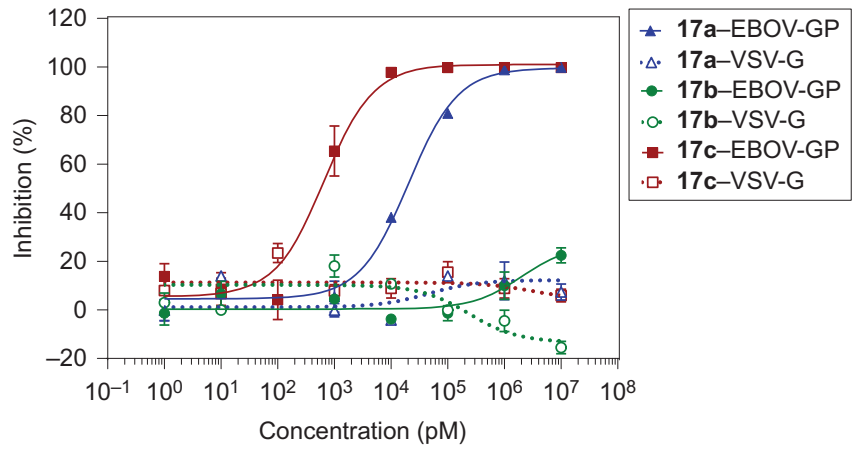

Figure 6 | Biological study of tridecafullerenes 17a-17c. The graphic shows the inhibition of infection with EBOV-GP- or VSV-GP-pseudotyped lentiviral particles of Jurkat DC-SIGN ${ }^{+}$cells using 17 a (blue), 17b (green) and 17c (red). In the cis-infection experiments, $2.5 \times 10^{5}$ Jurkat DC-SIGN ${ }^{+}$cells were challenged with 5,000 TCID of recombinant lentiviral particles. The results represent the mean of six independent experiments \pm s.e.m. Compounds 17a and 17c show a strong inhibitory activity when EBOV-GP-pseudotyped lentiviral particles are used. No inhibitory activity is detected when VSV-GPpseudotyped particles (control) are used as the infective agent. Compound $17 \mathrm{~b}$ (endowed with galactoses as carbohydrate units) does not show inhibitory activity because it is not able to block the DC-SIGN receptor.

low nanomolar concentrations, with an $\mathrm{IC}_{50}$ of $20.37 \mathrm{nM}(95 \%$ $\mathrm{CI}=14.63-28.37 \mathrm{nM})$. Compound $17 \mathrm{c}$ was almost one order of magnitude more potent at inhibiting the infection process, with an $\mathrm{IC}_{50}$ of $667 \mathrm{pM}$ (95\% CI $=411 \mathrm{pM}-1.08 \mathrm{nM}$ ) (Fig. 6).

Previous inhibition studies using the same infection model and fullerenes that displayed up to 36 mannoses show relative inhibitory potency (RIP) values at least two orders of magnitude smaller ${ }^{19}$. Moreover, huge virus-like particles (VLPs) with a radius of $16 \mathrm{~nm}$ and up to 1,640 mannoses $^{32}$ were 18 -fold less potent compared with compound $17 \mathrm{c}$ (see Table 1). These results confirm the efficiency of these systems both to interact with DC-SIGN and to compete with EBOV-GP-pseudotyped particles during their entry into target cells.

The cytotoxic effect of multivalent glycofullerenes was verified by a cell-proliferation assay using the Cell Titer 96 AQueous NonRadioactive Cell Proliferation Assay (Promega). There were no appreciable cytotoxic effects of compounds $17 \mathrm{a}-17 \mathrm{c}$ at the concentrations used in the infection experiments (Supplementary Fig. 6).

\section{Table 1 | Comparison of $I_{50}$ and RIP values of different mannosylated multivalent compounds.}

\begin{tabular}{|c|c|c|c|c|}
\hline Compound & $\begin{array}{l}\mathrm{IC}_{50} \\
(\mathrm{nM})\end{array}$ & $\begin{array}{l}\text { Mannoses } \\
\text { (No.) }\end{array}$ & RIP* $^{\star}$ & Reference \\
\hline 17c (120 mannoses) & 0.667 & 120 & $1.58 \times 10^{4}$ & This work \\
\hline $\operatorname{VLP}(1,620$ mannose $)$ & 0.91 & 1,620 & $8.6 \times 10^{2}$ & 33 \\
\hline 17a (120 mannoses) & 20.375 & 120 & $5.2 \times 10^{2}$ & This work \\
\hline VLP (540 mannoses) & 9.62 & 540 & $2.44 \times 10^{2}$ & 33 \\
\hline C60LL (36 mannoses) ${ }^{\dagger}$ & 300 & 36 & $1.17 \times 10^{2}$ & 20 \\
\hline C60 (36 mannoses) $)^{\dagger}$ & 68,000 & 36 & 0.5 & 20 \\
\hline C60 (12 mannoses) ${ }^{\dagger}$ & 2,000 & 12 & 53 & 20 \\
\hline$\alpha$-methyl-D- & $1.27 \times$ & 1 & 1 & 34 \\
\hline
\end{tabular}

Data obtained from inhibition studies using pseudotyped EBOV particles for the new compounds 17 a and 17c in comparison with other carbohydrate multivalent systems previously reported by us; ${ }^{*} \mathrm{RIP}$, calculated as $\left(\mathrm{IC}_{50}\right)$ mono/IC $\mathrm{C}_{50}{ }^{\star}$ valency $\left(\left(\mathrm{IC}_{50}\right)\right.$ mono, $\mathrm{I}_{50}$ of the monovalent compound; $I C_{50}{ }^{*}$ valency, $I C_{50}$ of the multivalent compound multiplied by the number of ligands present in the multivalent compound). ${ }^{\dagger}$ See Supplementary Fig. 5 for the chemical structure.

\section{Conclusions}

In summary, we synthesized giant globular multivalent glycofullerenes in which the central $\mathrm{C}_{60}$ core is covalently connected to 12 hexakis adducts of $\mathrm{C}_{60}$ to form the first tridecafullerenes reported so far. Each peripheral fullerene is endowed with ten monosaccharides and so a total of 120 carbohydrates decorate the periphery of each molecule. This represents the fastest dendrimer growth ever reported, affording molecular weights as high as $56 \mathrm{kDa}$.

The syntheses of non-symmetric hexakis adducts $(7,9,14 a, 14 b$, $15 \mathrm{a}$ and $\mathbf{1 5 b})$ as well as the tridecafullerenes $(17 \mathrm{a}-17 \mathrm{c})$ were accomplished efficiently by using CuAAC click-chemistry reactions. In this way, very sophisticated molecular ensembles were produced in a minimum of synthetic steps, and their apparent structural complexity does not place a limit for their application. Despite the high molecular weights, the new molecules were characterized by standard spectroscopic techniques (FTIR, ${ }^{1} \mathrm{H}$ NMR and ${ }^{13} \mathrm{C}$ NMR spectroscopy) as well as by DLS, XPS and TEM. Interestingly, NMR spectroscopy unambiguously revealed the high degree of symmetry $\left(\mathrm{T}_{\mathrm{h}}\right)$ in hexakis adducts.

Tridecafullerenes are soluble in water and, therefore, they were tested as globular multivalent systems to inhibit lectin-mediated viral infection processes in cellular assays. In particular, compounds $17 \mathrm{a}-17 \mathrm{c}$ were used to test their ability to inhibit the infection of cells 
by an artificial EBOV. These tridecafullerenes were found to block the EBOV infection efficiently in the subnanomolar concentration range. These values surpass by three orders of magnitude (two if the number of mannoses is considered) those exhibited by hexakis adducts endowed with 12 mannoses.

The aforementioned results reveal fullerenes to be appealing platforms for the study of multivalent interactions, particularly because of their biocompatibility and globular presentation. Furthermore, the compounds reported here pave the way to the introduction of dendritic dimers and trimers of monosaccharides as well as to the use of disaccharides to improve significantly the scope of the biological applications of tridecafullerenes.

\section{Methods}

Production of recombinant viruses. Recombinant viruses were produced in 293T cells. The viral construction was pseudotyped with the Zaire EBOV envelope GP or VSV-GP and expressed luciferase as a reporter of the infection ${ }^{29,33}$. One day (18-24 hours) before transfection, $5 \times 10^{6} 293 \mathrm{~T}$ cells were seeded onto $10 \mathrm{~cm}$ plates. The cells were cultured in DMEM medium supplemented with $10 \%$ heat-inactivated fetal bovine serum, $25 \mathrm{mg}$ gentamycin and $2 \mathrm{mM}$ L-glutamine. A few minutes before transfection, the medium on the transfection plates was changed to $9 \mathrm{ml} \mathrm{DMEM}$ and chloroquine was added to a $25 \mu \mathrm{M}$ final concentration. Transfection reactions with all the reagents at room temperature (r.t.) were prepared in $15 \mathrm{ml}$ tubes: $183 \mu \mathrm{l}$ of $2 \mathrm{M} \mathrm{CaCl}_{2}, 500 \mathrm{ng}$ of EBOV-GP or $2 \mu \mathrm{g}$ of VSV-GP, $21 \mu \mathrm{g}$ of pNL4-3 luc ${ }^{34}$ and $1,300 \mu \mathrm{l}$ of $\mathrm{H}_{2} \mathrm{O}$. Next, $1.5 \mathrm{ml}$ of $2 \times$ HBS (HEPES buffer saline) $\mathrm{pH} 7.00$ was added quickly to the tubes and bubbled for 30 seconds. HBS/DNA solution was gently dropped onto the medium. After eight hours of incubation at $37^{\circ} \mathrm{C}$ with $5 \% \mathrm{CO}_{2}$, the medium on the transfection plates was changed to $10 \mathrm{ml}$ DMEM and once again, one day after transfection, to $7 \mathrm{ml}$ DMEM. Transfection supernatants were harvested after 48 hours, centrifuged at 1,200 revolutions per minute for ten minutes at r.t. to remove cell debris and stored frozen at $-80^{\circ} \mathrm{C}^{32,33,35}$.

EBOV infection experiments. Infection was performed on Jurkat cells (T-lymphocyte cell line) that expressed the receptor DC-SIGN on their surfaces. As the EBOV does not infect T-lymphocytes, its entry is absolutely dependent on DC-SIGN for infection of the Jurkat cells ${ }^{29,36}$.

Jurkat-DC-SIGN cells $\left(2.5 \times 10^{5}\right)$ were plated into each well of a 96-well plate. Cells were incubated at r.t. for 20 minutes with the carbohydrate-based compounds and then challenged with 5,000 TCID (tissue culture infective dose) of recombinant viruses. After 48 hours of incubation, the cells were washed twice with PBS and assayed with the Luficerase Assay System (Promega).

The range of concentrations tested for compounds $17 \mathrm{a}-17 \mathrm{c}$ was $1 \mathrm{pM}$ to $10 \mu \mathrm{M}$. As a control, an experiment of infection with VSV-GP pseudoviruses was performed under the same conditions. Infection with VSV-GP was independent of the presence of DC-SIGN receptor.

Statistical analysis. The values of percentages of inhibition of the infection presented in Fig. 6 correspond to the mean of six independent experiments, and the error bars correspond to s.e.m. The $\mathrm{IC}_{50}$ values were estimated using $\mathrm{GraphPad}$ Prism v6.0 with a 95\% CI and settings for normalized dose-response curves.

Cytotoxicity assay. The Cell Titer 96 AQueous Non-Radioactive Cell Proliferation Assay (Promega) was used. Jurkat-DC-SIGN cells $\left(5 \times 10^{5}\right.$ cells per well) were seeded into the wells of 96-well plates and left to culture in the presence of different concentrations of glycofullerenes for the time of the infection assay. After 48 hours, the proliferation cell assay was performed. Briefly, $2 \mathrm{ml}$ of MTS (5-(3-carboxymethoxyphenyl)-2-(4,5-dimethylthiazolyl)-3-(4-sulfophenyl) tetrazolium) solution was mixed with $100 \mu$ of phenazine methosulfate (PMS) solution. The mix MTS/PMS in a volume of $20 \mu \mathrm{l}$ was pipetted into each well of the 96-well assay plate, which contained $100 \mu \mathrm{l}$ of cells in the culture medium. The plate was incubated for two hours at $37^{\circ} \mathrm{C}$ in a humidified $5 \% \mathrm{CO}_{2}$ atmosphere. After the incubation period, the absorbance at $490 \mathrm{~nm}$ was recorded using an enzyme-linked immunosorbent assay plate reader. As a control of the toxicity, the viability of the cells in the presence of $0.1 \%$ Triton-X100 was measured. The results of the assay are presented as the percentage of viable cells, which was calculated from the absorbance at $490 \mathrm{~nm}$ as compared with the absorbance shown by cells incubated without the addition of glycofullerenes, which represent $100 \%$ cell viability.

Received 17 December 2014; accepted 28 September 2015; published online 9 November 2015

\section{References}

1. Mammen, M., Choi, S.-K. \& Whitesides, G. M. Polyvalent interactions in biological systems: implications for design and use of multivalent ligands and inhibitors. Angew. Chem. Int. Ed. 37, 2754-2794 (1998).
2. Guo, Y. et al. Structural basis for distinct ligand-binding and targeting properties of the receptors DC-SIGN and DC-SIGNR. Nature Struct. Mol. Biol. 11, 591-598 (2004)

3. Imperiali, B. The chemistry-glycobiology frontier. J. Am. Chem. Soc. 134, 17835-17839 (2012)

4. Fasting, C. et al. Multivalency as a chemical organization and action principle. Angew. Chem. Int. Ed. 51, 10472-10498 (2012).

5. Imberty, A., Chabre, Y. M. \& Roy, R. Glycomimetics and glycodendrimers as high affinity microbial anti-adhesins. Chem. Eur. J. 14, 7490-7499 (2008).

6. Roy, R. A decade of glycodendrimer chemistry. Trends Glycosci. Glycotechnol. 15, 291-310 (2003).

7. Roy, R. \& Baek, M.-G. Glycodendrimers: novel glycotope isosteres unmasking sugar coding. Case study with T-antigen markers from breast cancer MUC1 glycoprotein. Rev. Mol. Biotechnol. 90, 291-309 (2002).

8. Chabre, Y. M. \& Roy, R. in Advances in Carbohydrate Chemistry and Biochemistry Vol. 63 (ed. Derek, H.) 165-393 (Academic Press, 2010).

9. Cecioni, S., Imberty, A. \& Vidal, S. Glycomimetics versus multivalent glycoconjugates for the design of high affinity lectin ligands. Chem. Rev. 115, 525-561 (2015).

10. Hirsch, A. \& Vostrowsky, O. $\mathrm{C}_{60}$ hexakisadducts with an octahedral addition pattern-a new structure motif in organic chemistry. Eur. J. Org. Chem. 2001, 829-848 (2001)

11. Lamparth, I., Maichle-Mössmer, C. \& Hirsch, A. Reversible template-directed activation of equatorial double bonds of the fullerene framework: regioselective direct synthesis, crystal structure, and aromatic properties of Th- $\mathrm{C}_{66}(\mathrm{COOEt})_{12}$ Angew. Chem. Int. Ed. Engl. 34, 1607-1609 (1995).

12. Hirsch, A. in Fullerenes and Related Structures (ed. Hirsch, A.) 1-65 (Topics in Current Chemistry 199, Springer, 1999).

13. Iehl, J., Pereira de Freitas, R., Delavaux-Nicot, B. \& Nierengarten, J.-F. Click chemistry for the efficient preparation of functionalized [60]fullerene hexakisadducts. Chem. Commun. 2450-2452 (2008).

14. Nierengarten, J.-F. et al. Fullerene sugar balls. Chem. Commun. 46, 3860-3862 (2010).

15. Sánchez-Navarro, M., Muñoz, A., Illescas, B. M., Rojo, J. \& Martín, N. [60]Fullerene as multivalent scaffold efficient molecular recognition of globular glycofullerenes by concanavalin A. Chem. Eur. J. 17, 766-769 (2011).

16. Rísquez-Cuadro, R., García Fernández, J. M., Nierengarten, J.-F. \& Ortiz Mellet, C. Fullerene-s $p^{2}$-iminosugar balls as multimodal ligands for lectins and glycosidases: a mechanistic hypothesis for the inhibitory multivalent effect. Chem. Eur. J. 19, 16791-16803 (2013).

17. Cecioni, S. et al. Synthesis of dodecavalent fullerene-based glycoclusters and evaluation of their binding properties towards a bacterial lectin. Chem. Eur. J. 17, 3252-3261 (2011).

18. Nierengarten, I. \& Nierengarten, J.-F. Fullerene sugar balls: a new class of biologically active fullerene derivatives. Chem. Asian J. 9, 1436-1444 (2014).

19. Durka, M. et al. The functional valency of dodecamannosylated fullerenes with Escherichia coli FimH-towards novel bacterial antiadhesives. Chem. Commun. 47, 1321-1323 (2011).

20. Luczkowiak, J. et al. Glycofullerenes inhibit viral infection. Biomacromolecules 14, 431-437 (2013).

21. Hörmann, F. \& Hirsch, A. Giant fullerene polyelectrolytes composed of $C_{60}$ building blocks with an octahedral addition pattern and discovery of a new cyclopropanation reaction involving dibromomalonates. Chem. Eur. J. 19, 3188-3197 (2013)

22. Wasserthal, L. K., Kratzer, A. \& Hirsch, A. Sequential fullerenylation of bismalonates-efficient access to oligoclusters with different fullerene building blocks. Eur. J. Org. Chem. 2013, 2355-2361 (2013).

23. Balbinot, D. et al. Electrostatic assemblies of fullerene-porphyrin hybrids: toward long-lived charge separation. J. Phys. Chem. B 107, 13273-13279 (2003).

24. Wessendorf, F. et al. Implementation of a Hamilton-receptor-based hydrogenbonding motif toward a new electron donor-acceptor prototype: electron versus energy transfer. J. Am. Chem. Soc. 129, 16057-16071 (2007).

25. Durka, M. et al. The inhibition of liposaccharide heptosyltransferase WaaC with multivalent glycosylated fullerenes: a new mode of glycosyltransferase inhibition. Chem. Eur. J. 18, 641-651 (2012).

26. Ciampi, S. et al. Functionalization of acetylene-terminated monolayers on $\mathrm{Si}$ (100) surfaces: a click chemistry approach. Langmuir 23, 9320-9329 (2007).

27. Collman, J. P., Devaraj, N. K., Eberspacher, T. P. A. \& Chidsey, C. E. D. Mixed azide-terminated monolayers: a platform for modifying electrode surfaces. Langmuir 22, 2457-2464 (2006).

28. Devaraj, N. K., Decreau, R. A., Ebina, W., Collman, J. P. \& Chidsey, C. E. D. Rate of interfacial electron transfer through the 1,2,3-triazole linkage. J. Phys. Chem. B 110, 15955-15962 (2006).

29. Alvarez, C. P. et al. C-type lectins DC-SIGN and L-SIGN mediate cellular entry by Ebola virus in cis and in trans. J. Virol. 76, 6841-6844 (2002).

30. Kondratowicz, A. S. et al. T-cell immunoglobulin and mucin domain 1 (TIM-1) is a receptor for Zaire Ebolavirus and Lake Victoria Marburgvirus. Proc. Natl Acad. Sci. USA 108, 8426-8431 (2011). 
31. Carette, J. E. et al. Ebola virus entry requires the cholesterol transporter Niemann-Pick C1. Nature 477, 340-343 (2011).

32. Ribeiro-Viana, R. et al. Virus-like glycodendrinanoparticles displaying quasiequivalent nested polyvalency upon glycoprotein platforms potently block viral infection. Nature Commun. 3, 1303 (2012).

33. Yang, S. L. et al. Generation of retroviral vector for clinical studies using transient transfection. Hum. Gene Ther. 10, 123-132 (1999).

34. Connor, R. I., Chen, B. K., Choe, S. \& Landau, N. R. Vpr is required for efficient replication of Human-Immuno-Deficiency-Virus Type-1 in mononuclear phagocytes. Virology 206, 935-944 (1995).

35. Luczkowiak, J. et al. Pseudosaccharide functionalized dendrimers as potent inhibitors of DC-SIGN dependent Ebola pseudotyped viral infection. Bioconjugate Chem. 22, 1354-1365 (2011).

36. Lasala, F., Arce, E., Otero, J. R., Rojo, J. \& Delgado, R. Mannosyl glycodendritic structure inhibits DC-SIGN-mediated Ebola virus infection in cis and in trans. Antimicrob. Agents Chemother. 47, 3970-3972 (2003).

\section{Acknowledgements}

Financial support by the European Research Council (ERC-2012-ADG_320441 (Chirallcarbon), ITN-2008-213592 (CARMUSYS)), Ministerio de Economía y Competitividad (MINECO) of Spain (projects CTQ2014-52045-R, CTQ2011-23410 and

CTQ2012-31914), the Comunidad Autónoma de Madrid (PHOTOCARBON project
S2013/MIT-2841), Instituto de Salud Carlos III (ISCIII) (FIS PI1101580 and FIS1400708), the Agence National de la Recherche (ANR, Programme Blanc 2011), the International Center for Frontier Research in Chemistry and LabEx 'Chimie des Systèmes Complexes' is acknowledged. N.M. thanks the Alexander von Humboldt Foundation. S.P.V. and K.B. thank FNRS (FRIA fellowship).

\section{Author contributions}

A.M., D.S., I.N., M.H. and K.B. carried out the synthesis and characterization of all the new derivatives. L.R.-P. and J.-S.R. realized and analysed the DLS and TEM. L.R.-P. realized the XPS analyses and contributed to the writing of the paper. J.L. and R.D. realized the biological and cytotoxicity studies. B.M.I., S.P.V., J.R., R.D., J.-F.N. and N.M. designed the project, supervised the work, discussed the data and wrote the manuscript.

\section{Additional information}

Supplementary information and chemical compound information are available in the online version of the paper. Reprints and permissions information is available online at www.nature.com/reprints. Correspondence and requests for materials should be addressed to J.R., R.D., J.F.N. and N.M.

\section{Competing financial interests}

The authors declare no competing financial interests. 